\title{
Excitation and dynamics in the extended bose-hubbard model
}

\author{
Benoît Grémaud ${ }^{1,2,3,4}$ and G.G. Batrouni ${ }^{5,6,1,2}$ \\ ${ }^{1}$ MajuLab, CNRS-UNS-NUS-NTU International Joint Research Unit UMI 3654, Singapore \\ ${ }^{2}$ Centre for Quantum Technologies, National University of Singapore, 3 Science Drive 2, Singapore 117543, Singapore \\ ${ }^{3}$ Department of Physics, National University of Singapore, 2 Science Drive 3, Singapore 117542, Singapore \\ ${ }^{4}$ Laboratoire Kastler Brossel, UPMC-Sorbonne Universités, CNRS, \\ ENS-PSL Research University, Collège de France, 4 Place Jussieu, 75005 Paris, France \\ ${ }^{5}$ INLN, Université de Nice-Sophia Antipolis, CNRS; 1361 route des Lucioles, 06560 Valbonne, France \\ ${ }^{6}$ Institut Universitaire de France, 103, Boulevard Saint-Michel, 75005 Paris, France
}

(Dated: November 6, 2018)

\begin{abstract}
The one-dimensional extended bosonic Hubbard model has been shown to exhibit a variety of phases ranging from Mott insulator and superfluid to exotic supersolids and Haldane insulators depending on the filling and the relative value of the contact $(U)$ and near neighbor $(V)$ interaction strengths. In this paper we use the density matrix renormalization group and the time evolving block decimation numerical methods to study in detail the dynamics and excitation spectra of this model in its various phases. In particular, we study in detail the behavior of the charge and neutral gaps which characterize the Mott, charge density and Haldane insulating phases. We also show that in addition to the gapless modes at $k=0$, the supersolid phase exhibits gapless modes at a finite $k$ which depends on the filling.
\end{abstract}

\section{INTRODUCTION}

The bosonic Hubbard model (BHM) has continued to attract interest since its introduction by Fisher et al. 11. This interest stems from its use to understand many physical phenomena such as the effect of disorder on superfluids and the appearance of the compressible Bose glass phase [1], quantum phase transitions between strongly correlated exotic phases etc. Interest in the BHM intensified with the experimental realization of Bose-Einstein condensates and the ability to load them in optical lattices 2. Under experimentally realizable conditions, these systems are described by the BHM and its extensions [3] with highly tunable parameters and in one, two and three dimensions which makes them ideal for studying quantum phase transitions and exotic phases in strongly correlated systems.

Increasingly, over the last several years, the physics of strongly correlated quantum systems has focused on the existence and properties of unconventional phases and phase transitions. In addition to well studied Mott insulating behavior caused by strong on-site repulsion at commensurate filling, extensive quantum Monte Carlo (QMC) simulations have shown that a strong enough near neighbor repulsion can lead to insulating incompressible density wave order (CDW) at integer and half odd integer fillings. Topological phases, such as the Haldane insulator which is a gapped phase characterized by a non-local (string) order parameter [4, 5] can be also found in the extended BHM [6] in one dimension. Finally, doping these phases can lead to phase separation or to supersolid (SS) phases 9222 .

Even though the phase diagram of the extended BHM is now well understood, the excitation spectra of the various ground states have been less studied [23, 24], essentially because the numerical methods providing the ground state properties, such as exact diagonalization or
QMC, become limited in the dynamical domain. More recently, for quasi-1D systems, the extension of the density matrix renormalization group method (DMRG) to the time domain or, equivalently, the time evolving density matrix method (TEBD) have proved to be extremely successful in probing the dynamical properties of the system, thereby providing reliable excitation spectrum [25]27]. In this paper, we extend our work in 8,9 to study the excitation spectrum of the one dimensional extended BHM in different phases, namely the Mott insulating phase (MI), the Haldane insulating phase (HI), the charge density wave phase (CDW) and the supersolid phase (SS).

The paper is organized as follows. In section II we present the model and the various methods to compute the ground state properties and excitation spectrum. In section III, we present the dependence of the dynamical structure factor on the near neighbor repulsion, $V$, at fixed filling, $n=1$, and fixed value of the contact repulsion, $U$ and hopping, $t$. In section [IV] we study the dynamical structure factor in the SS phase for different fillings and explain its main properties using a mapping of the extended BHM to the Heisenberg model for a spin $1 / 2$ chain in a finite magnetic field. In addition, we describe the evolution of the spectrum across the SS-SF transition. In section $\mathrm{V}$, we discuss the qualitative differences found in the dynamical structure factor in the phase which is obtained by underdoping the half-filled CDW and in the SS phase. A summary of results and conclusions is in section VI. 


\section{MODEL AND METHODS}

\section{A. The model Hamiltonian}

The one dimensional extended BHM we shall study is described by the Hamiltonian,

$$
\begin{aligned}
H= & -t \sum_{i}\left(a_{i}^{\dagger} a_{i+1}+a_{i+1}^{\dagger} a_{i}\right)+\frac{U}{2} \sum_{i} n_{i}\left(n_{i}-1\right) \\
& +V \sum_{i} n_{i} n_{i+1} .
\end{aligned}
$$

The sum over $i$ extends over the $L$ sites of the lattice, periodic boundary conditions were used in the QMC simulations 28, and open conditions in the DMRG and the TEBD simulations. The onsite repulsive interaction energy, $U$, is put equal to unity and sets the energy scale. The time scale being given by $\hbar / U$, it is also put equal to unity. The operator $a_{i}\left(a_{i}^{\dagger}\right)$ destroys (creates) a boson on site $i, n_{i}=a_{i}^{\dagger} a_{i}$ is the number operator on site $i, t$ is the hopping amplitude and $V$ is the near neighbor repulsive interaction parameter. Since we will typically study the system in the canonical ensemble, we did not include a chemical potential term in $H$.

The charge gap is given by,

$$
\begin{aligned}
\Delta_{c}(n) & =\mu(n)-\mu(n-1) \\
& =E_{0}(n+1)+E_{0}(n-1)-2 E_{0}(n)
\end{aligned}
$$

where the chemical potential is given by $\mu(n)=E_{0}(n+$ $1)-E_{0}(n)$ and $E_{0}(n)$ is the ground state energy of the system with $n$ particles and is obtained both with QMC and DMRG. The neutral gap, $\Delta_{n}$, is obtained using DMRG by targeting the lowest excitation with the same number of bosons. For the CDW and HI phases, the chemical potentials at both ends are set to (opposite) large enough values, when using DMRG, such that the ground state degeneracy and the low energy edge excitations are lifted 6, 8, 24, 29].

For a bosonic filling $\bar{n}$ close to unity, the Bose-Hubbard model can be reasonably approximated by the AF spin-1 Heisenberg model:

$$
H_{S}=\sum_{i} J\left(S_{i}^{x} S_{i+1}^{x}+S_{i}^{y} S_{i+1}^{y}\right)+\lambda S_{i}^{z} S_{i+1}^{z}+D\left(S_{i}^{z}\right)^{2}
$$

where $\lambda$ is the axial anisotropy and $D$ the ion anisotropy. One has the following mapping between the parameters [7]: $J=-t \bar{n}, \lambda=V$ and $D=U / 2$.

\section{B. Time Evolving Block Decimation}

As mentioned above, the excitation spectra are obtained using the TEBD, first in imaginary time to obtain the ground state, then in real time to compute the density-density correlation function. In each case, we have used a number preserving algorithm. We have checked that the ground state properties (energy, site density, double occupancy...) obtained with the TEBD exactly match the properties of the ground state obtained from the DMRG, using the ALPS library [27]. The space and time correlation functions $\left\langle A_{i}(T) B_{j}\right\rangle$, where $\langle\cdots\rangle$ is the ground state average and where $A_{i}(T)$ is the time evolution of the operator $A_{i}$ in the Heisenberg picture, have been obtained by writing,

$$
\left\langle A_{i}(T) B_{j}\right\rangle=e^{i E_{G S} T}\left\langle G S\left|A_{i} e^{-i H T} B_{j}\right| G S\right\rangle,
$$

where $E_{G S}$ is the ground state energy. Therefore, computing the correlation function can be done as follows:

- From the matrix product state (MPS) representation of the ground state, $|G S\rangle$, one computes the MPS of the initial state $|\Psi(0)\rangle=B_{j}|G S\rangle$.

- The state $|\Psi(0)\rangle$ is evolved using the real time TEBD, providing the MPS of $|\Psi(T)\rangle$, and thereby allowing the computation of the correlation function as $\left\langle G S\left|A_{i}\right| \Psi(T)\right\rangle$.

In what follows, we focus on the density-density correlation, i.e. $A_{i}=B_{i}=n_{i}-\left\langle n_{i}\right\rangle$, more precisely, the initial state consists of creating a density excitation in the middle of the chain: $B_{j}|G S\rangle$ with $j=L / 2$. We then compute $\left\langle A_{i}(T) B_{j}\right\rangle$ for all sites and times $T$ up to 150 , with a time step equal to 0.1 (a smaller timestep was actually used for the propagation). Finally, the dynamical structure factor $S(k, \omega)$ is computed from the Fourier transform of the density-density correlation with respect to $i-j$ and $T$. In order to smooth out oscillations caused by the finite time window, we actually compute the Fourier transform of $\left\langle A_{i}(T) B_{j}\right\rangle \exp \left(-4 T^{2} / T_{\max }^{2}\right)$.

\section{MOTT-HALDANE-CDW TRANSITIONS}

The qualitative description of the different phases is based on the Heisenberg model EQ.4. More precisely, the phases are characterized by the values of the string order parameters:

$$
O^{\alpha}=\lim _{|i-j| \rightarrow \infty}\left\langle S_{i}^{\alpha} e^{i \pi \sum_{p=i+1}^{j-1} S_{p}^{\alpha}} S_{j}^{\alpha}\right\rangle
$$

characterizing a loose antiferromagnetic order along the different axes $\alpha=x, y, z$. They are associated with an underlying non-local discrete $Z_{2} \otimes Z_{2}$ symmetry of the Heisenberg model, Eq. (4) 30. In the large- $D$ phase (the MI state for bosons), the $O^{\alpha}$ vanish. In the Haldane phase, the two discrete $Z_{2}$ symmetries are broken, resulting in nonvanishing string order $O^{\alpha}$. Finally, in the Ising phase (the CDW for bosons), only the $Z_{2}$ symmetry along the $z$-axis is broken such that only the string order $\mathrm{O}^{z}$ is non-vanishing. Note that in that phase, the string order and the antiferromagnetic order (the CDW order for bosons) are equivalent [34. 
The lowest elementary excitations from the ground state $\left(k a=0, S_{z}=0\right)$ are part of a triplet, one neutral $\epsilon^{(0)}(k)\left(\delta N=S_{z}=0,\right)$, two charge ones $\epsilon^{( \pm)}(k)$ $\left(\delta N=S_{z}= \pm 1\right)$ [23, 35, where $\delta N$ corresponds to change in the total number of bosons. In each sector, one defines a gap which corresponds to the minimum of the elementary excitations over all $k$ values: $G^{(0)}$ and $G^{( \pm)}$.

The minima are located either at $k a=0$ or $k a=\pi$. From linear response theory, the structure factor reads

$$
S(k, \omega) \propto \sum_{m} \frac{|\langle k, m|\delta \hat{n}| G S\rangle|^{2}}{\omega+i \eta+E_{G S}-E_{k, m}},
$$

where $|k, m\rangle$ denotes the different excited states of $H$ for a given momentum $k$, and $E_{k, m}$ the corresponding energy. For single excitations, one simply has $E_{k, m}=$ $\epsilon^{(m)}(k)$. Doubly excited states for fixed $k$ are made of two single excitations: $\left|q, m ; k-q, m^{\prime}\right\rangle$, corresponding to an energy $\epsilon^{(m)}(q)+\epsilon^{\left(m^{\prime}\right)}(k-q)$.

By definition, the charge gap of the system is $\Delta_{c}=$ $G^{(+)}+G^{(-)}$, i.e. the minimum energy for adding a particle plus the minimum energy for removing a particle (increasing or decreasing $S_{z}$, in the Heisenberg model). The neutral gap corresponds to the minimum of either the elementary neutral excitations, i.e. $G^{(0)}$, or of $\epsilon^{(+)}(k-q)+\epsilon^{(-)}(q)$, i.e., a combination of two charge excitations. Since, the minimum of both the elementary charge excitation $\epsilon^{( \pm)}(q)$ is attained at either $q=0$ or $q=\pi$, the minimum of the two-particle excitation necessarily takes place at $k=0$ and has the value $G^{(+)}+G^{(-)}$, and corresponds then to the lower bound of a two-particle continuum. In short, the neutral gap value is given by the minimum of $G^{(0)}$ and $G^{(+)}+G^{(-)}$.

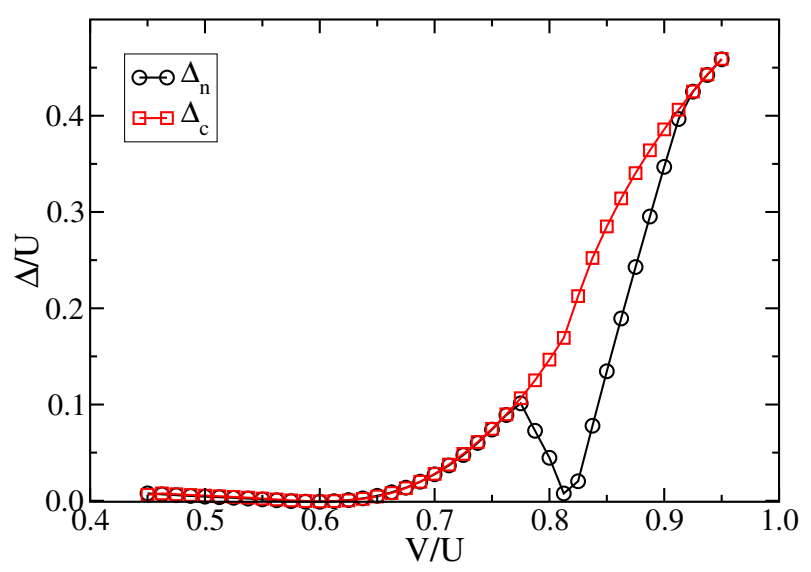

Figure 1. Mott - Haldane - CDW transitions at fixed $t / U=$ 0.25 . Around $V_{C} \approx 0.75 U$, the neutral and charge gaps start to differ, indicating that $\left.G^{(} 0\right)<G^{(+)}+G^{(-)}$: the gap for the single particle neutral excitations is smaller than the gap for the two particle excitations. The fact that only the neutral gap vanishes at the HI-CDW transition is a signature that the string order $O^{z}$ along the $z$-axis remains finite, where $O^{x}$ and $O^{y}$ orders vanish.

It is well-known that in the Haldane phase, the neutral gap changes from one type to the other [6, 23, 35] and is emphasized in Fig. 1, where around $V_{C} \approx 0.75 U$, the neutral and the charge gaps start having different values. For lower $V$ values, one has $\Delta_{n}=\Delta_{c}=G^{(+)}+G^{(-)}$, whereas for larger $V$ values, one has $G^{(0)}=\Delta_{n}<\Delta_{c}=$ $G^{(+)}+G^{(-)}$. This results from the fact that, in the Haldane phase, the elementary excitations are all gapped, with a minimum occuring at $k a=\pi$ 23, 35]. For a fixed value of $U$, corresponding to a fixed value of the ion anisotropy, $D$, in the corresponding spin Hamiltonian, $G^{( \pm)}$increases with increasing $V$ (i.e. $\lambda$ ), whereas $G^{(0)}$ decreases. This can be understood by starting at the Heisenberg point, $(D=0, \lambda=J)$, where, due to $S U(2)$ invariance of the spin Hamiltonian, all single particle excitation energies are the same, so that $G^{(0)}=G^{( \pm)}$. Increasing the ion anisotropy, $D$, i.e. going toward the Mott Phase (or decreasing $\lambda$ ) gives rise to a smaller in-plane gap (i.e. the elementary charge gap) $G^{( \pm)}<G^{(0)}$.

This evolution of the neutral and charge gaps can be seen in the behavior of the structure factor $S(k, \omega)$. We emphasize that even though the structure factor $S(k, \omega)$ is a neutral excitation, i.e. conserves the total number of bosons, it also couples to the two-particle continuum composed of elementary charge excitations $\epsilon^{(+)}(k-q)+\epsilon^{(-)}(q)$. As explained above, in the limit $k \rightarrow 0$, the minimum energy corresponds to the charge gap $\Delta_{c}=G^{(+)}+G^{(-)}$, such that even if we expect $S(k, \omega)$ to vanish at $k a=0$, the value for $\Delta_{c}$ can be obtained by extrapolating the behavior of $S(k, \omega)$ around $k a=0$.

In the Mott phase, top Fig. 2, one can clearly see that the gap at $k a=\pi$ is much larger than the gap at $k a=0$. Note that the gap $k a=\pi$ differs from $G^{(0)}$, since, in the MI phase, $\epsilon^{(0)}(k)$ is minimum at $k a=0$ and maximum at $k a=\pi$. The neutral and the charge gap have the same value $G^{(+)}+G^{(-)}$, which can be obtained from $S(k, \omega)$ by extrapolating the gap value to $k a=0$. At the Mott-Haldane transition, bottom Fig. 2. $S(k, \omega)$ exhibits (almost) gapless excitation around $k a=0$, whereas the excitation is cleary gapped at $k a=\pi$. Since the transition corresponds to breaking both hidden $Z_{2}$ symmetries, both the neutral and the charge gaps vanish, corresponding to vanishing elementary charge excitations gap $G^{( \pm)}$, but a finite elementary neutral excitation gap $G^{(0)}$. 

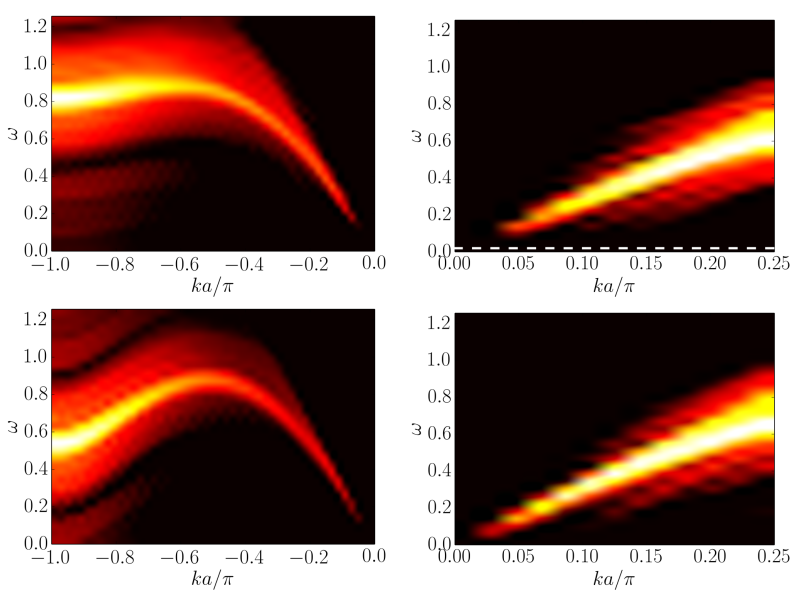

Figure 2. TEBD excitation spectra. TOP: $V / U=0.4$, $t / U=0.25 \mathrm{MI}$ phase. The gap at $k a=\pi$ is much larger than the gap at $k a=0 . \Delta_{c}=\Delta_{n}=S(k \rightarrow 0, \omega)$. The white dashed line in the right panel shows the value of the gap (small but non-vanishing). BOTTOM: $V / U=0.58, t / U=0.25$ at the MI-HI transition. The excitations are almost gapless near $k a=0$, whereas the excitation is cleary gapped at $k a=\pi$. See text.
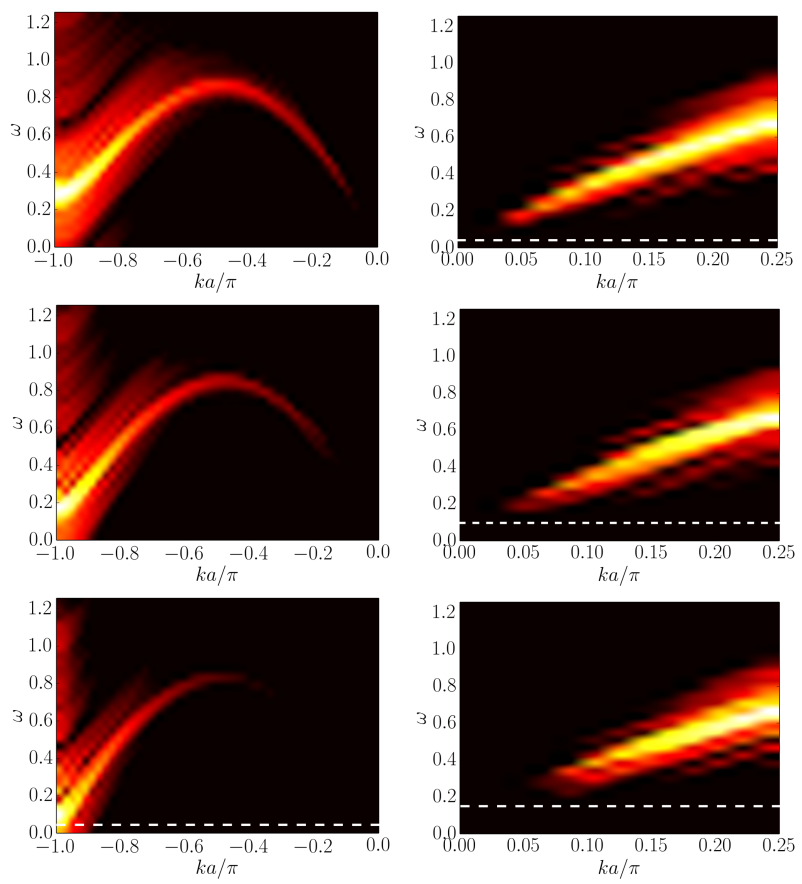

Figure 3. TEBD excitation spectra inside the HI phase. TOP: $V / U=0.7, t / U=0.25$, the gap at $k a=\pi$, i.e. $G^{(0)}$, decreased while the gap at $k a=0$ is nonvanishing, $\Delta_{n}=\Delta_{c}=G^{(+)}+G^{(-)} \neq 0$. The white dashed line gives the value of the gap. MIDDLE: $V / U=0.75, t / U=0.25$ inside the HI phase at the symmetric point. The gap at $k a=\pi, G^{(0)}$, has the same value as the gap at $k a=0$, $G^{(+)}+G^{(-)}$, indicated by the white dashed line. BOTTOM: $V / U=0.79, t / U=0.25$ inside the HI phase. The gap at $k a=\pi, \Delta_{n}=G^{(0)}$, is smaller than the charge gap at $k a=0$, $\Delta_{c}=G^{(+)}+G^{(-)}$. The white dashed line in the left plot corresponds $\Delta_{n}=G^{(0)}$, whereas, in the right plot, it corresponds to $\Delta_{c}=G^{(+)}+G^{(-)}$.

Inside the Haldane phase, but for a value $V<V_{c}$, top Fig. 3 . we see that the gap at $k a=\pi$, i.e. $G^{(0)}$, has decreased whereas the gap at $k a=0$, i.e. $\Delta_{n}=\Delta_{c}=$ $G^{(+)}+G^{(-)}$, is non vanishing. At the value $V \approx V_{c}$, middle Fig. 3, the gap at $k a=\pi, G^{(0)}$, has almost the same value as the gap at $k a=0, G^{(+)}+G^{(-)}$. When $V>V_{c}$, bottom Fig. 3, we see that one is now in the opposite situation: $\Delta_{n}=G^{(0)}$ is clearly smaller than $\Delta_{c}=G^{(+)}+G^{(-)}$.

At the transition between the Haldane phase and the charge density wave phase, top Fig. 4. $\Delta_{n}=G^{(0)}$ vanishes while the charge gap at $k a=0, G^{(+)}+G^{(-)}$remains finite. This corresponds to the fact that across the transition, the hidden $Z_{2}$ symmetry along the $z$-axis remains broken, whereas the hidden $Z_{2}$ symmetry along in the $X Y$ plane is restored. Since the string order $O^{z}$ is broken by charge excitations but left invariant under neutral excitations, i.e. commutes with the $S_{i}^{z}$ operators, only the charge gap is protected by the finite value of the order $O^{z}$ and remains finite at the transition. On the other hand, since the string order $O^{x}$, which is broken by neutral excitations (i.e. applying $S_{i}^{z}$ ), vanishes at the 
transition, the neutral gap has to close at the transition.

Finally, in the CDW phase, bottom Fig. 4 both the neutral and the charge gap increase, but still having $\Delta_{n}=G^{(0)}<G^{(+)}+G^{(-)}=\Delta_{c}$.
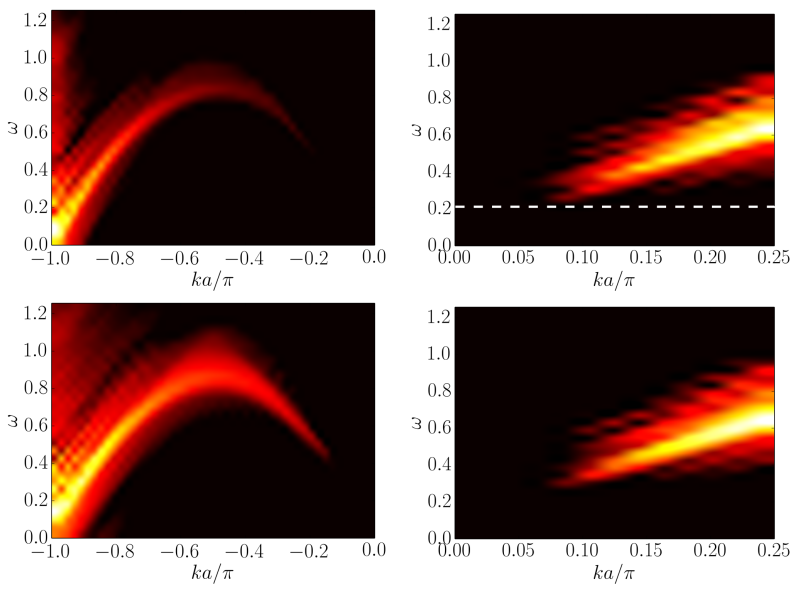

Figure 4. TEBD excitation spectra. TOP: $V / U=0.82$, $t / U=0.25$ at the HI-CDW transition, At $k a=\pi, \Delta_{n}=$ $G^{(0)}=0$ whereas $\Delta_{c}=G^{(+)}+G^{(-)}$at $k a=0$, remains finite and is given by the white dashed line in the right plot. BOTTOM: $V / U=0.84, t / U=0.25$ in the CDW phase, the neutral gap at $k a=\pi$ is finite, but has smaller value than the charge gap: $\Delta_{n}=G^{(0)}<G^{(+)}+G^{(-)}=\Delta_{c}$.

Finally, in Fig. 5. we show the excitation spectrum when adding a boson to the system, i.e. corresponding to the operators $A_{i}=b_{i}$ and $B_{j}=b_{j}^{\dagger}$ in Eq. (5). In both plots, the large vertical offset corresponds to the chemical potential for adding a boson, $\mu^{+}$. By definition, the value of the charge gap is $\mu^{+}-\mu^{-}$, where $\mu^{-}$is the chemical potential for removing a boson. Therefore the minimum of the excitation spectrum can be written $\bar{\mu}+\Delta_{c} / 2$, where $\bar{\mu}=\left(\mu^{+}+\mu^{-}\right) / 2$ is the average chemical potential. The parameters for the top plot are the same as in Fig. 2](top panels), i.e. the Mott Phase. One clearly sees that the minimum of the charge excitation is obtained at $k a=0$, the value at $k a=\pi$ being much larger. The bottom plot corresponds to bottom panels Fig. 3 i.e. the Haldane phase where the neutral and charge gaps are different. The minimum of the excitation occurs at $k a=\pi$, whereas around $k a=0$, one has a two-particle continuum, made of one neutral excitation and one charge excitation, the minimum value, at $k a=0$, being $\mu^{+}+G^{(0)}$, i.e. $\Delta_{c} / 2+$ $G^{(0)}$.
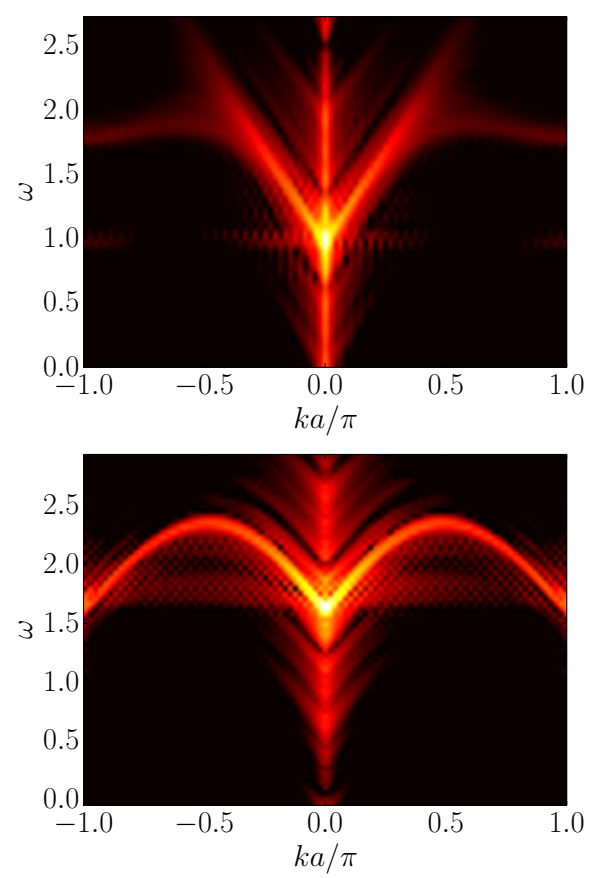

Figure 5. Excitation spectra obtained when adding a boson to the system, i.e. corresponding to the operators $A_{i}=b_{i}$ and $B_{j}=b_{j}^{\dagger}$ in Eq. (5). In both plots, the vertical offset corresponds to the chemical potential $\mu_{+}$for adding a boson. The parameters for the top plot are the same as in Fig.2 (top panels), i.e. the Mott Phase. One clearly see that the minimum of the charge excitation is obtained at $k a=0$, the value at $k a=\pi$, being much larger. The bottom plot corresponds to bottom panels Fig. 3 i.e. the Haldane phase where the neutral and the charge gap are different. The lowest excitation occurs at $k a=\pi$. Around $k a=0$, one has a two-particle continuum, made of one neutral excitation and one charge excitation.

\section{SUPERSOLID PHASE}

The hallmark of the supersolid phase is the presence of both a long range diagonal (density) order and superfluidity. A typical density profile, obtained using DMRG for $U=1, V=0.75$ and $t=0.2$, is shown in Fig. 6. The oscillations of the density between 0.25 and 2.25 , around the average value $n=1.25$ signal long range density order but which, nonetheless, is not in the CDW phase since the average density, $n=1.25$, is not commensurate. 


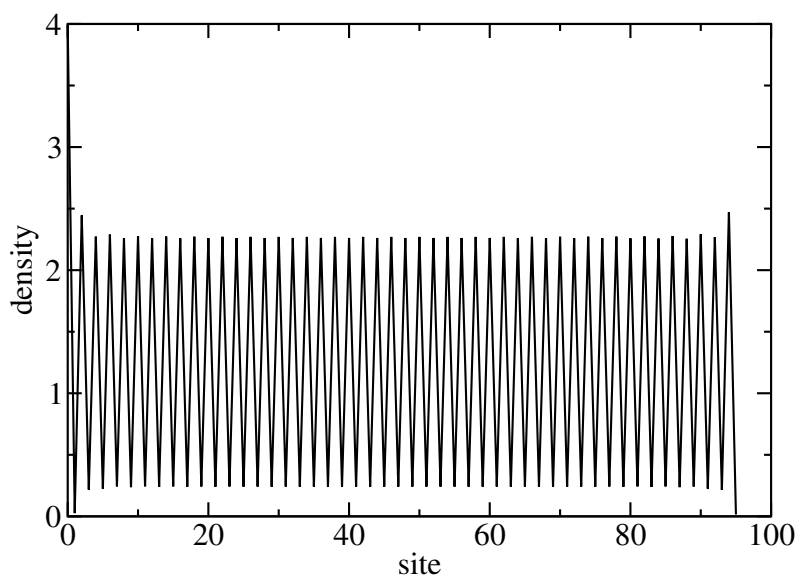

Figure 6. Density profile in the supersolid phase $U=1$, $V=0.75$ and $t=0.2, n=1.25$. The density oscillates between, 0.25 and 2.25 , indicating long range density order at the incommensurate average density $n=1.25$.

Figures 7, 8, 9 and 10 show the the dispersion for several values of the doping: $n=1.25, n=1.167, n=1.125$ and $n=1.08333$ respectively. All data were obtained for $L=96$ sites, $U=1, V=0.75, t=0.2$ and the system is in the supersolid phase.

As expected in the supersolid phase, the system exhibits gapless excitations at $k=0$ and $k=\pi$, but one clearly sees additional gapless excitations at a momentum $k_{S S}$ that depends on the density. It turns out that the value of $k_{S S}$ is in excellent agreement with the value $2 \pi \delta n$ (see below), where $\delta_{n}=n-1$, i.e. $k a=\pi / 2$ $(k a=\pi / 3, k a=\pi / 4, k a=\pi / 6)$ for $\delta_{n}=1 / 4\left(\delta_{n}=1 / 6\right.$, $\left.\delta_{n}=1 / 8, \delta_{n}=1 / 12\right)$.

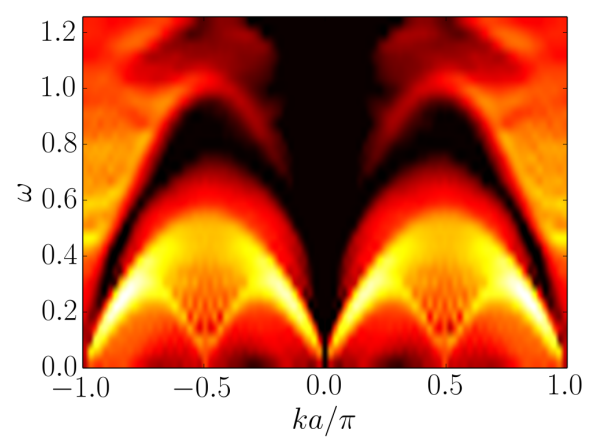

Figure 7. $t=0.2, n=1.25$. The gapless excitations at $k a=0$ emphasize the superfluid nature of the phase. The lower part of the excitation spectrum has a periodicity $\pi / a$ reflecting the $2 a$ periodicity of the low energy effective hamiltonian, which is an AF spin $1 / 2$ chain (see text). The additional gapless mode at $k a=0.5 \pi$ corresponds to the gapless mode at $2 k a=\pi$ of the effective AF chain.

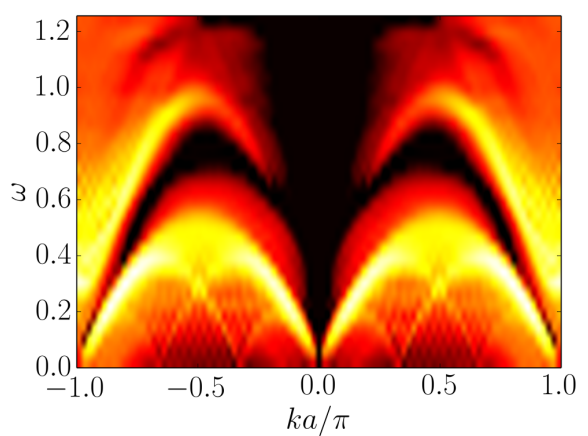

Figure 8. $t=0.2, n=1.167$. As in Fig. 7 the periodicity of the lower part of the excitation spectrum can be understood from the low energy effective hamiltonian, which is an AF spin $1 / 2$ chain in a finite magnetic field. The additional gapless mode at $k a=\pi / 3$ corresponds to the low energy incommensurate modes of the spin chain at a finite magnetization.

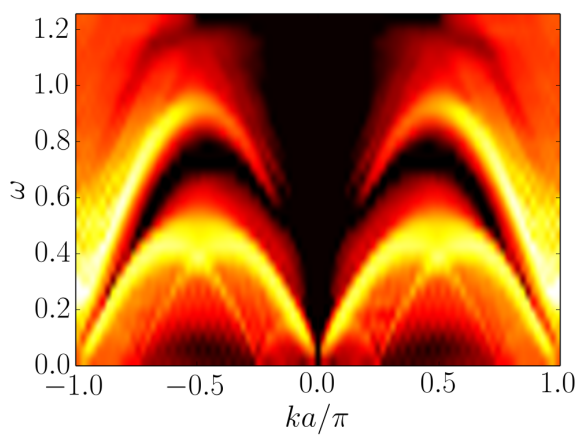

Figure 9. $t=0.2, n=1.125$. As in Fig. 8. The additional gapless mode at $k a=\pi / 4$ corresponds to the low energy incommensurate modes of a spin- $1 / 2$ chain at a finite magnetization.

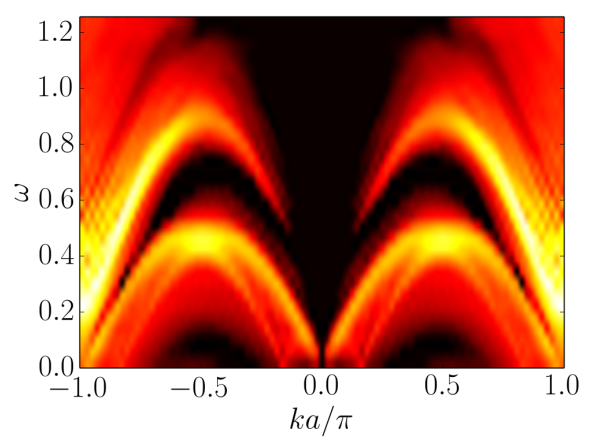

Figure 10. $t=0.2, n=1.08333$. As in Fig. 8 The additional gapless mode at $k a=\pi / 6$ corresponds to the low energy incommensurate modes of a spin- $1 / 2$ chain at a finite magnetization. 


\section{A. Effective Spin-1/2 Heisenberg model of the supersolid}

We consider the situation where the supersolid phase has a density $n=5 / 4$, see Fig. 6. This occurs when one dopes the system in the CDW phase, i.e. for $V>U / 2$. The density pattern, in the limit $U \gg t$, obtained from both the DMRG and the QMC computations show that the ground state has a (nearly) vanishing density on alternate sites and that the other states are (almost) built on either the $n=2$ or $n=3$ Fock states. We, therefore, expect the low energy excitation to be given by an effective spin-1/2 Heisenberg model, where we map $|3\rangle(|2\rangle)$ to $|\uparrow\rangle(|\downarrow\rangle)$, and remove the state with vanishing density. The new chain has therefore an effective lattice spacing equal to $2 a$. The effective interaction arises from the virtual hopping of the bosons to the empty sites. Taking into account the different intermediate states, one obtains the following effective spin-half Heisenberg hamiltonian:

$H_{\mathrm{eff}}=\sum_{i} \frac{J_{\mathrm{eff}}}{2}\left(S_{i}^{+} S_{i+1}^{-}+S_{i}^{-} S^{-}{ }_{i+1}\right)+\lambda_{\mathrm{eff}} S_{i}^{z} S_{i+1}^{z}-B_{\mathrm{eff}} S_{i}^{z}$,

where

$$
\begin{aligned}
& J_{\text {eff }}=-t^{2}\left(\frac{3}{4 V-2 U}\right) \\
& \lambda_{\text {eff }}=2 t^{2}\left(\frac{3}{4 V-2 U}+\frac{2}{4 V-U}-\frac{3}{5 V-2 U}-\frac{2}{3 V-U}\right) \\
& B_{\text {eff }}=2 \delta \mu+2 t^{2}\left(\frac{3}{5 V-2 U}-\frac{2}{3 V-U}\right),
\end{aligned}
$$

where $\delta \mu=\mu-2 U . \quad \lambda_{\text {eff }}$ is positive for a large range of $(V, U)$ values, so that the preceding Hamiltonian corresponds essentially the AF spin-half Heisenberg model in a magnetic field (the negative sign of $J_{\text {eff }}$ can be removed through the mapping $\left.\left(S_{x}, S_{y}\right) \rightarrow-\left(S_{x}, S_{y}\right)\right)$.

Defining the ratio $\rho=2 V / U$, one obtains:

$$
\begin{aligned}
& J_{\text {eff }}=-\frac{t^{2}}{U}\left(\frac{3}{2 \rho-2}\right) \\
& \lambda_{\text {eff }}=\frac{2 t^{2}}{U}\left(\frac{3}{2 \rho-2}+\frac{2}{2 \rho-1}-\frac{6}{5 \rho-4}-\frac{4}{3 \rho-2}\right) \\
& B_{\text {eff }}=2 \delta \mu+4 t^{2}\left(\frac{3}{5 \rho-4}-\frac{2}{3 \rho-2}\right) .
\end{aligned}
$$

For $B_{\text {eff }}=0$, the system is in the $A F(X Y)$ phase when $\lambda_{\text {eff }}<\left|J_{\text {eff }}\right|,\left(\lambda_{\text {eff }}>\left|J_{\text {eff }}\right|\right)$ 36, 37. From the preceding expressions, the ratio $\Delta=\lambda_{\text {eff }} /\left|J_{\text {eff }}\right|$ starts from the value $\Delta=2$ at $\rho=1$ and then decreases. The isotropic point $\Delta=1$ is crossed around $\rho \approx 1.15$, such that for $V=0.75 U$, i.e. $\rho=1.5$, the system is the $X Y$ phase. The ground state has therefore a vanishing magnetization, corresponding to an average density $n=5 / 4$. For $t=0$, this corresponds to $\delta \mu=0$, i.e. to the boundary between the $n=1$ and $n=3 / 2 \mathrm{CDW}$. Then, for any finite $B_{\text {eff }}$, the average magnetization is positive (negative) corresponding to an average density larger (less) than $5 / 4$. In addition, the effective spin correlations exhibit spatial oscillations whose period depends on the magnetization, which, in turn, leads to gapless excitations at finite momentum. More precisely, starting from the correlation functions of the spin- $1 / 2$ chain obtained using the bosonisation approach [37] and taking into account that $J_{\text {eff }}$ is negative and that the lattice spacing is $2 a$, one can show that both the in-plane $\left(\left\langle S^{+} S^{-}\right\rangle\right)$and the out-ofplane $\left(\left\langle S^{z} S^{z}\right\rangle\right)$ correlations yield oscillations corresponding to a wavevector $k_{0} a=\pi(m+1 / 2)$, where $m$ is the magnetization. The latter is related to the average density $\bar{n}$ of the Bose-Hubbard model: $m=2 \bar{n}-5 / 2$, such that $m+1 / 2=2(\bar{n}-1)=2 \delta n$, where $\delta n=\bar{n}-1$. Therefore, the gapless excitations correspond to $k_{0} a=2 \pi \delta n$, in perfect agreement with Figs. 7, 8, 9 and 10

Finally, one can see in Figs. 7, 8, 9 and 10 that the effective period of the lowest part of the excitation spectrum is $\pi / 2 a$, corresponding the doubling of the lattice spacing. This is not true for the higher excitations which are gapped and most likely involving the empty sites.

\section{B. SS-SF transition}

We have also studied the evolution of the structure factor $S(k, \omega)$ across the supersolid-superfluid transition, at fixed density and interaction strengths, increasing the hopping amplitude from $t / U=0.24$ (SS) to $t / U=0.3$ (SF).

\section{TEBD results}

From the density plots, see Fig. 11 (top), Fig. 12 (top) and Fig. 13 (top), we see that the SS-SF transition is driven by the disappearance of the spatial modulation. This behavior is also predicted by the standard meanfield theory where the ground state of the system is assumed to be a tensor product of onsite wavefunctions (see below).

The disappearance of the spatial modulation results in an opening of the gap at $k a=\pi$, see Fig. 13 (bottom), which is well described by the mean-field theory, since it only amounts to a change of the spatial periodicity, i.e. from $2 a$ to $a$, of the effective Hamiltonian 38 .

On the other hand this simple mean-field cannot capture the long range quantum correlations that lead to the gapless modes at $k a=k_{S S} a=2 \pi \delta n$ and the mapping to the spin- $1 / 2$ is no longer valid close to the transition since one cannot neglect previously empty sites. From that point of view, the exact fate of these gapless mode is still lacking a physical explanation. 

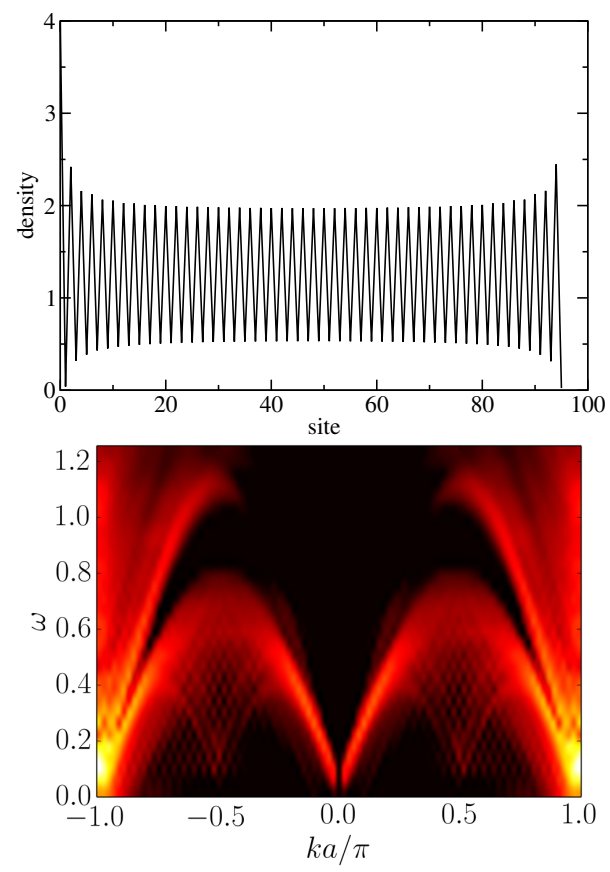

Figure 11. The SS phase at $U=1, V=0.75$ and $t=0.24$, $n=1.25$. Top: Density profile: The CDW order is still almost perfect. Bottom: The gapless modes at $k a=0.5 \pi$ are still visible, but their contributions to $S(k, \omega)$ have a smaller weight when compared with Fig. 7
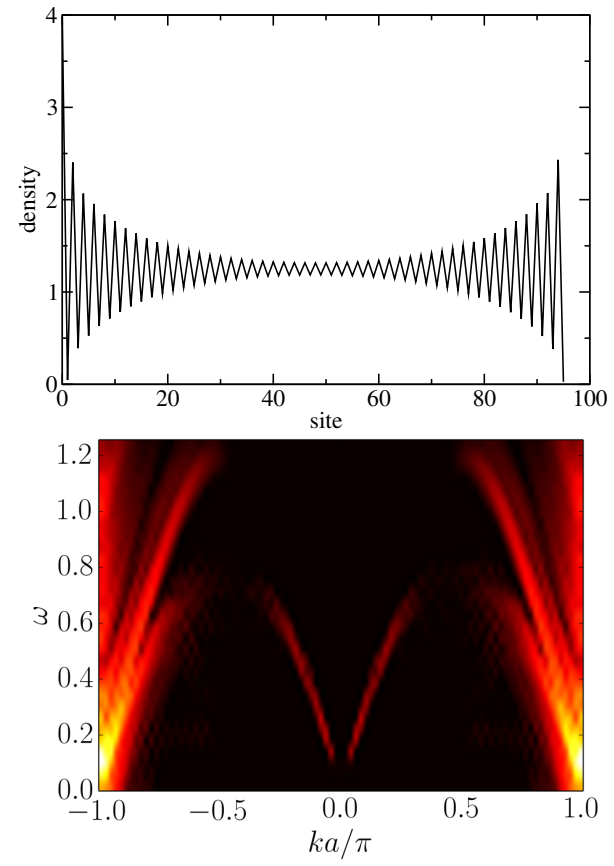

Figure 12. The supersolid phase at $U=1, V=0.75$ and $t=$ $0.26, n=1.25$. Top: The density pattern does not show a well defined CDW. Bottom: The gapless mode at $k a=0.5 \pi$ has almost disappeared, but the system is still gapless at $k a=\pi$
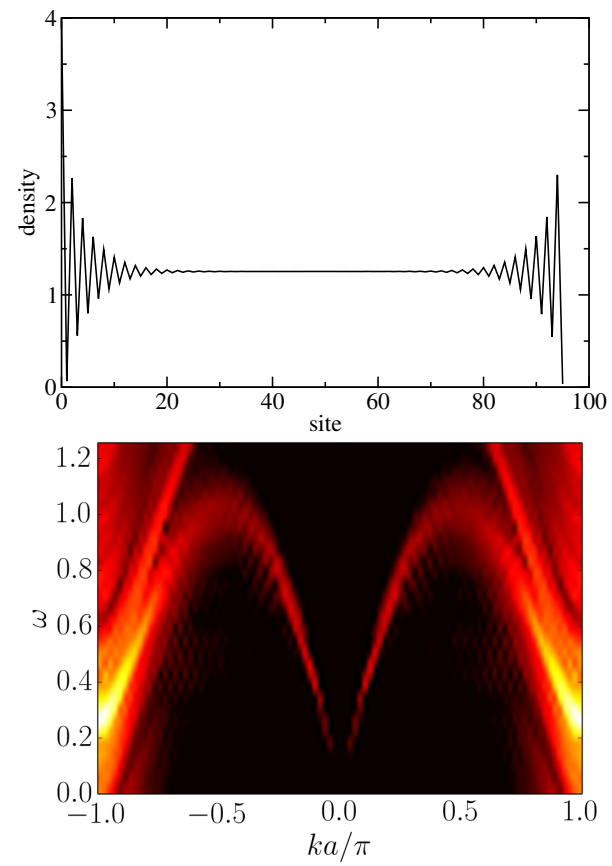

Figure 13. The superfluid phase at $U=1, V=0.75$ and $t=0.3, n=1.25$. Top: The density profile no longer shows CDW pattern. Bottom: The only gapless mode is at $k a=0$, as expected in the SF phase; at $k a=\pi$, the system is now gapped,

\section{Mean-field results}

As explained above, a well known mean-field method to solve the Bose-Hubbard model is the Gutzwiller ansatz, where the ground state wavefunction is assumed to be a tensor product of onsite wavefunctions:

$$
|\Psi\rangle=\bigotimes_{i}\left|\psi_{i}\right\rangle \text { where }\left|\psi_{i}\right\rangle=\sum_{n=0}^{N_{\max }} f_{n, i}|n, i\rangle .
$$

$|n, i\rangle$ represents the Fock state of $n$ atoms occupying the site $i, n_{\max }$ is a cut off in the maximum number of atoms per site, and $f_{n, i}$ is the probability amplitude of having the site $i$ occupied by $n$ atoms.

Minimizing the mean-field energy $\langle\Psi|H| \Psi\rangle$ over the $f_{n, i}$ allows us to determine the mean-field ground state properties as functions of the different parameters $(U, t, V, \mu)$. For instance, the superfluid phase corresponds to a non vanishing value of the order parameter $\langle\Psi|b| \Psi\rangle$, whereas the Mott phase corresponds to a vanishing order parameter and the $\psi_{i}$ are pure Fock states. In the CDW phase, the order parameter $\langle b\rangle$ vanishes; the density, $\langle n(k a=0)\rangle$ and the staggered density, $\langle n(k a=\pi)\rangle$, have the same value. The supersolid phase corresponds to non-vanishing values for both $\langle b(k a=0)\rangle$ and $\langle b(k a=\pi)\rangle$; the density still exhibits oscillations at $k a=\pi$. The superfluid phase corresponds to a homogeneous density and only the $k a=0$ order parameter $\langle b(k a=0)\rangle$ has a non-vanishing value. 
We present mean-field results for $U=1, V=1.5$ and $\mu=1.8$. Note that since the chemical potential is fixed, the density changes as $t / U$ is changed. Figure 14 shows the different quantities as functions of $t / U$. For $0 \leq t \leq 0.25$, the system is in the CDW insulating phase: The order parameter $\langle b\rangle$ vanishes and the density and staggered density have the same value; the CDW corresponds to a density pattern $\cdots 2020202020 \cdots$. For $0.25 \leq t \leq 1.26$ the system is in the supersolid phase: Both $\langle b(k=0)\rangle$ and $\langle b(k a=\pi)\rangle$ are non-vanishing. The density still presents oscillations at $k a=\pi$. For $t \geq 1.25$, the system is in the usual superfluid phase.

The mean field approach also allows us to compute the excitation spectrum. Since in both the CDW and SS phases the periodicity of the ground state is $2 a$, the spectrum is defined in the reduced Brillouin zone $[-\pi / 2 a, \pi / 2 a]$ and has two branches. In the CDW phase, the elementary excitations are gapped, as expected. In the SS phase, the lower branch becomes gapless with a linear behavior around $k=0$. At the SS-SF transition, the periodicity of the ground state goes back to $a$, so that the two elementary excitation branches merge at $k a=\pi / 2$.

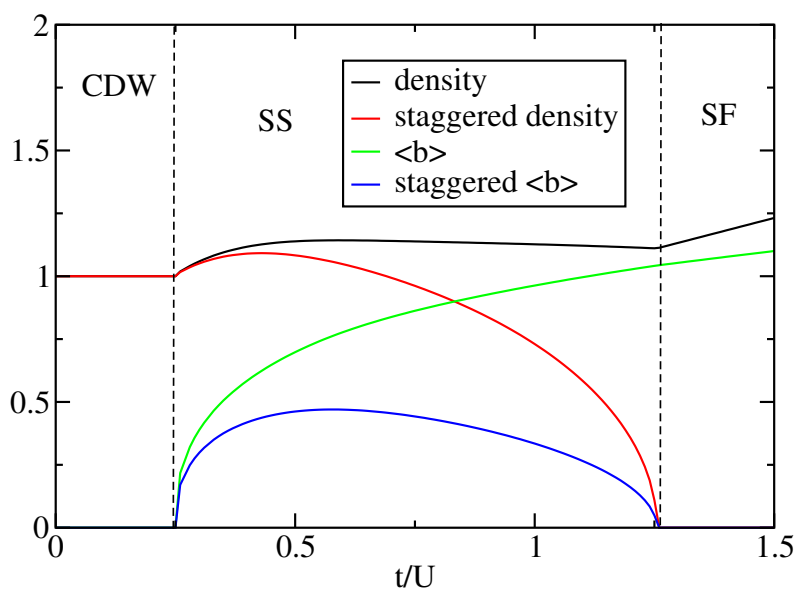

Figure 14. (Color online) Mean field phase diagram for $U=$ $1, V=1.5$ and $\mu=1.8$ as a function of $t$. For $0 \leq t \leq$ 0.25 , the system is in a CDW insulating phase: the order parameter $\langle b\rangle$ vanishes and the density and staggered density have the same value. The CDW corresponds to a density pattern $\cdots 2020202020 \cdots$. For $0.25 \leq t \leq 1.26$ the system is in the supersolid phase: both $\langle b(k=0)\rangle$ and $\langle b(k a=\pi)\rangle$ are non-vanishing. The density still presents oscillations at $k a=\pi$. For $t \geq 1.25$, the system is superfluid.

\section{UNDERDOPED HALF-FILLING CDW}

In this section, we compare the structure factor obtained in the SS phase with the one for the phase between the half-filled CDW and the superfluid phase, see Fig. 15. A typical density profile is shown in Fig. 16, the parameters are $U=1, V=0.75$ and $t=0.1$, corresponding to an average density $n=0.4375$. One clearly sees that the density pattern is different from the one in the supersolid phase: the long wavelength modulation of the CDW is a signature of a vanishing DLRO, in contrast to the SS phase. On the other hand, one has an overall power law decay of the ODLRO $g(x) \propto x^{-1 / 2 K}$, but with a coefficient $K<1 / 2$ emphasizing that the SF can be localized with a single impurity [24]. The difference with the SS phase also appears in the structure factor, Fig. 17. One has only two gapless modes, one at $k a=0$ and one at $k a \approx 0.8 \pi$, but the excitations at $k=\pi$ are gapped. Therefore, the periodicity of the lower part of the spectrum is just $2 \pi / a$ and not $\pi / a$ as in the SS phase.

Since, at very low values of $t$, the density pattern for the half-filled CDW phase is $\cdots 01010101 \cdots$, there is a natural mapping onto a spin-half AF Heisenberg model with a vanishing total magnetization: $|0\rangle \rightarrow|\downarrow\rangle$ and $|1\rangle \rightarrow|\uparrow\rangle$. The underdoped CDW phase corresponds then to a non vanishing total magnetization $S_{z} \approx n-1 / 2$. However, contrary to the SS phase, there is no simple way to get the effective $J$ and $\lambda$ coefficients: the initial state $\cdots 01000101 \cdots$ and a state after one hopping $\cdots 01001001 \cdots$ are actually degenerate in the limit $t \rightarrow 0$, thereby preventing a proper expansion of the Bose-Hubbard Hamiltonian. Nevertheless, one can still argue that the gapless mode at $k a \approx 0.8 \pi$ corresponds to the incommensurate gapless mode appearing in the spinspin correlation function for the spin-half AF Heisenberg model in a finite magnetic field. In particular the value $k_{0} a=0.8 \pi$ is compatible with the bosonisation prediction $2 \pi n$ [37.

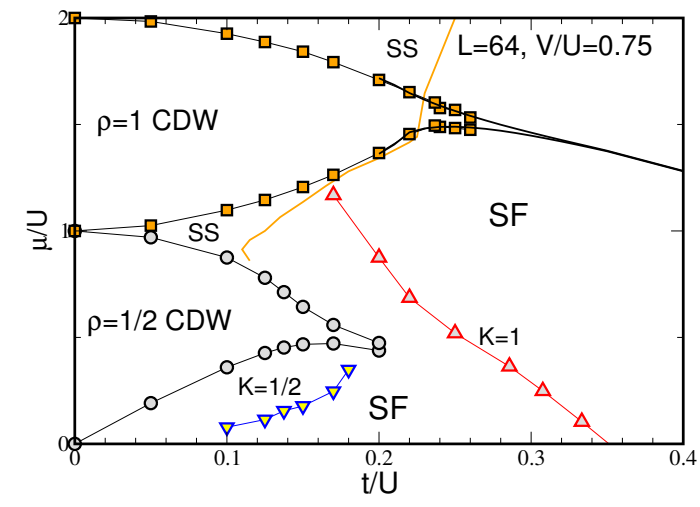

Figure 15. (color online) Detail of the $n=1 / 2$ lobe (from QMC) where we also determined the constant $K$ lines for $K=1,1 / 2$. 


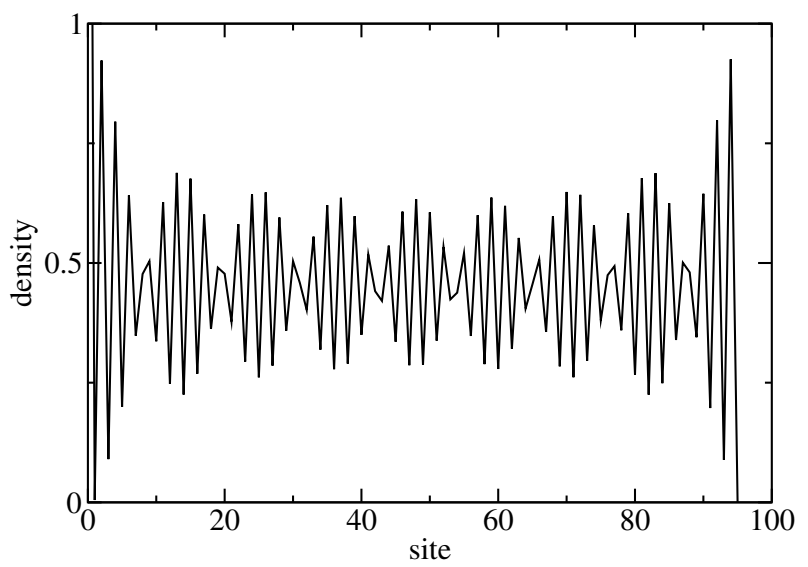

Figure 16. Density profile in the underdoped $1 / 2 \mathrm{CDW}$ phase $U=1, V=0.75$ and $t=0.1, n=0.4375$. The overall modulation of the density emphasizes the difference with the SS phase.
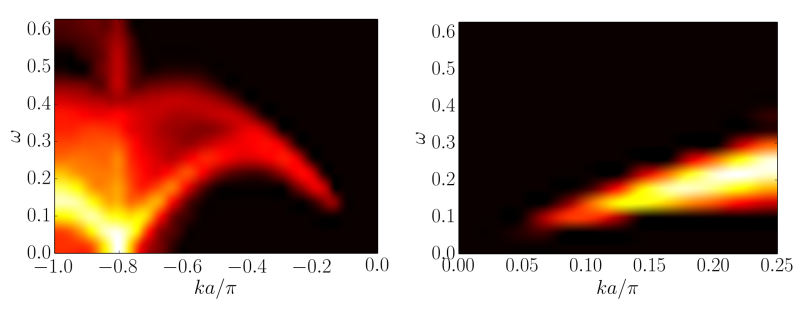

Figure 17. (color online) Structure factor in the underdoped CDW: $U=1, V=0.75 t=0.2$, the density is $n=0.40625$. The gapless mode at $k a=0$ indicates the ODLRO. The gapless mode at $k a \approx 0.8 \pi$ is compatible with the bosonisation prediction $2 \pi n$ for an $\mathrm{AF}$ spin- $1 / 2$ chain in a finite magnetic field.

\section{CONCLUSIONS}

In summary, we have studied the excitation spectra of the extended Bose-Hubbard model. Along, the MI-HICDW transition, the dynamical structure factor exhibits behavior similar to the spin-spin correlation for the $S=1$ Heisenberg model. For instance, it shows a difference between the neutral and charge gaps in the HI phase.

In the SS phase, we have shown that the system has additional gapless modes at a $k$ value that depends on the average density of the system. They can be mapped to the incommensurate gapless modes of an AF spin-1/2 chain at finite magnetization. They are a signature of the DLRO present in the SS phase. These modes fade away when moving towards the SF phase, and, in addition, a gapped mode appears at $k a=\pi$, marking the disappearance of the DLRO.

Finally, we have shown that underdoping the CDW at half-filling, the excitation spectrum differs from the one in the SS phase, emphasizing that even though the system exhibits superfluidity and oscillations in the density, there is no DLRO.

\section{ACKNOWLEDGMENTS}

The Centre for Quantum Technologies is a Research Centre of Excellence funded by the Ministry of Education and National Research Foundation of Singapore.
[1] M.P.A. Fisher, P. B. Weichman, G. Grinstein and D. S. Fisher, Phys. Rev. B40, 546 (1989).

[2] M. Greiner, O. Mandel, T. Esslinger, T. W. Hänsch and I. Bloch, Nature 415, 39 (2002).

[3] D. Jaksch, H.-J. Briegel, J. I. Cirac, C. W. Gardiner and P. Zoller, Phys. Rev. Lett. 82, 1975 (1999).

[4] M. den Nijs and K. Rommelse, Phys. Rev. B40, 4709 (1989).

[5] F. D. M. Haldane, Phys. Lett. 93A, 464 (1983); Phys. Rev. Lett. 50, 1153 (1983).

[6] E. G. Dalla Torre, E. Berg and E. Altman, Phys. Rev. Lett. 97, 260401 (2006).

[7] E. Berg, E. G. Dalla Torre, T. Giamarchi and E. Altman, Phys. Rev. B77, 245119 (2008).

[8] G.G. Batrouni, R.T. Scalettar, V. G. Rousseau and B. Grémaud, Phys. Rev. Lett. 110, 265303 (2013).

[9] G. G. Batrouni, V. G. Rousseau, R. T. Scalettar, and B. Grmaud Phys. Rev. B 90, 205123 (2014).

[10] G. G. Batrouni, R. T. Scalettar, G. T. Zimanyi and A. P. Kampf, Phys. Rev. Lett. 74, 2527 (1995).

[11] G. G. Batrouni and R. T. Scalettar, Phys. Rev. Lett. 84,
1599 (2000).

[12] K. Góral, L. Santos and M. Lewenstein, Phys. Rev. Lett. 88, 170406 (2002).

[13] S. Wessel and M. Troyer, Phys. Rev. Lett. 95, 127205 (2005).

[14] M. Boninsegni and N. Prokof'ev, Phys. Rev. Lett. 95, 237204 (2005).

[15] P. Sengupta, L. P. Pryadko, F. Alet, M. Troyer and G. Schmid, Phys. Rev. Lett. 94, 207202 (2006).

[16] A. van Otterlo, K-H. Wagenblast, R. Baltin, C. Bruder, R. Fazio and G. Schön, Phys. Rev. B52, 16176 (2005).

[17] G.G. Batrouni, F. Hébert and R.T. Scalettar, Phys. Rev. Lett. 97, 087209 (2006).

[18] S. Yi, T. Li and C. P. Sun, Phys. Rev. Lett. 98, 260405 (2007)

[19] T. Suzuki and N. Kawashima, Phys. Rev. B75, 180502(R) (2007).

[20] L. Dang, M. Boninsegni and L. Pollet, Phys. Rev. B78, 132512 (2008).

[21] L. Pollet, J. D. Picon, H. P. Büchler and M. Troyer, Phys. Rev. Lett. 104, 125302 (2010). 
[22] B. Capogrosso-Sansone, C. Trefzger, M. Lewenstein, P. Zoller and G. Pupillo, Phys. Rev. Lett. 104, 125301 (2010).

[23] O. Golinelli, Th. Jolicoeur, and R. Lacaze, J. Phys.: Condens. Matter 5, 1399 (1993).

[24] T.D. Kühner, S.R. White, and H. Monien, Phys. Rev. B61, 12474 (2000).

[25] G. Vidal, Phys. Rev. Lett. 91, 147902 (2003);ibid. 93, 040502 (2004).

[26] U. Schollwock Annals of Physics 326, 96 (2011).

[27] B. Bauer et al. (ALPS collaboration), J. Stat. Mech. P05001 (2011).

[28] V.G. Rousseau, Phys. Rev. E77, 056705 (2008); ibid. E78, 056707 (2008); V.G. Rousseau and D. Galanakis, arXiv:1209.0946.

[29] D. Rossini and R. Fazio, New J. Phys. 14, 065012 (2012).

[30] I. Affeck, T. Kennedy, E.H. Lieb, and H. Tasaki, Phys.
Rev. Lett. 59, 799 (1987).

[31] T. Kennedy, and H. Tasaki, Commun. Math. Phys. 147, 431 (1992).

[32] O. Golinelli, Th. Jolicoeur, and R. Lacaze Phys. Rev. B 45, 9798 (1992).

[33] R. Botet, R. Jullien, and M. Kolb, Phys. Rev. B 28, 3914 (1983).

[34] M. Oshikawa, J. Phys.: Condens. Matter 4, 7469 (1992).

[35] G. Fth and J. Slyom, J. Phys.: Condens. Matter 5, 8983 (1993).

[36] H-J. Mikeska, A. Kolezhuk, in Quantum magnetism, edited by U. Schollwck, J. Richter, D. Farnell, and R. Bishop (Springer Berlin Heidelberg, 2004).

[37] T. Giamarchi, Quantum Physics in One Dimension, (Oxford Science Publications, 2004).

[38] M. Iskin, Phys. Rev. A83, 051606(R) (2011).

[39] R.T. Scalettar, G. G. Batrouni, A.P. Kampf, and G. T. Zimanyi Phys. Rev. B 51, 8467 (1995). 